\title{
Relação entre o Helicobacter pylori e a doença do refluxo gastroesofágico: uma revisão integrativa
}

\author{
Relationship between Helicobacter pylori and gastroesophageal reflux disease: an \\ integrative review
}
Relación entre Helicobacter pylori y la enfermedad por reflujo gastroesofágico: una revisión integradora

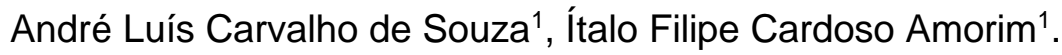

\begin{abstract}
RESUMO
Objetivo: Discutir sobre a possível influência do Helicobacter pylori como um fator determinante na Doença do Refluxo Gastroesofágico (DRGE) e suas possíveis complicações. Métodos: Trata-se de uma Revisão Integrativa de Literatura na qual utilizou-se as bases de dados NCBI, PubMed e SciELO. Os descritores escolhidos foram "Helicobacter pylori", "refluxo gastroesofágico", "esfíncter esofágico inferior". Assim, 15 artigos entre 2018 a 2021 foram escolhidos para revisão. Resultados: A infecção por $H$. pylori produz alterações no pH estomacal e, considerando que a DRGE causa sintomas associados repercussão da acidez do conteúdo gástrico no esôfago, a terapia de erradicação pode, além de corrigir o pH, gerar melhora nos sintomas de refluxo. Por outro lado, essa relação pode não existir ou a infecção pode até gerar uma piora do quadro de DRGE, dependendo de diversos fatores genéticos e ambientais. Considerações finais: Devem ser realizados novos estudos a respeito da influência da infecção por $H$. pylori na DRGE, principalmente no Ocidente, devido à alta prevalência das duas patologias, a fim de que sejam feitas as melhores condutas em pacientes com doenças gastroesofágicas.
\end{abstract}

Palavras-chave: Helicobacter pylori, Refluxo gastroesofágico, Esfíncter esofágico inferior.

\begin{abstract}
Objective: Discuss the possible influence of Helicobacter pylori as a determining factor in Gastroesophageal Reflux Disease (GERD) and its possible complications. Methods: This is an Integrative Literature Review in which the NCBI, PubMed and SciELO databases were used. The descriptors chosen were "Helicobacter pylori", "gastroesophageal reflux", "lower esophageal sphincter". Thus, 15 articles from 2018 to 2021 were chosen for review. Results: $H$. pylori infection produces changes in stomach $\mathrm{pH}$ and, considering that GERD causes symptoms associated with repercussions of acidic gastric contents in the esophagus, eradication therapy can, in addition to correcting the $\mathrm{pH}$, improve reflux symptoms. On the other hand, this relationship may not exist or the infection may even worsen the GERD picture, depending on several genetic and environmental factors. Final considerations: Further studies on the influence of $H$. pylori infection on GERD should be carried out, especially in the West, due to the high prevalence of the two pathologies, so that the best management is done in patients with gastroesophageal diseases.
\end{abstract}

Keywords: Helicobacter pylori, Gastroesophageal reflux, Lower esophageal sphincter.

\section{RESUMEN}

Objetivo: Discutir la posible influencia de Helicobacter pylori como factor determinante en la Enfermedad por Reflujo Gastroesofágico (ERGE) y sus posibles complicaciones. Métodos: Se trata de una Revisión de

\footnotetext{
${ }^{1}$ Faculdades Santo Agostinho (FASA), Vitória da Conquista - BA. *E-mail: andrecarvalholcs98@gmail.com
} 
Literatura Integrativa en la que se utilizaron las bases de datos NCBI, PubMed y SciELO. Los descriptores elegidos fueron "Helicobacter pylori", "reflujo gastroesofágico", "esfínter esofágico inferior". Así, se eligieron para revisión 15 artículos de 2018 a 2021. Resultados: La infección por $H$. pylori produce cambios en el pH del estómago y, considerando que la ERGE ocasiona síntomas asociados a repercusiones del contenido ácido gástrico en el esófago, la terapia de erradicación puede, además de corregir el $\mathrm{pH}$, mejorar los síntomas del reflujo. Por otro lado, esta relación puede no existir o la infección puede incluso empeorar el cuadro de ERGE, dependiendo de varios factores genéticos y ambientales. Consideraciones finales: Se deben realizar más estudios sobre la influencia de la infección por $H$. pylori en la ERGE, especialmente en Occidente, debido a la alta prevalencia de las dos patologías, con el fin de realizar el mejor manejo de los pacientes con enfermedades gastroesofágicas.

Palabras clave: Helicobacter pylori, Reflujo gastroesofágico, Esfínter esofágico inferior.

\section{INTRODUÇÃO}

A Doença do Refluxo Gastroesofágico (DRGE) é um distúrbio de evolução crônica, de alta prevalência em países ocidentais, em que o conteúdo gástrico reflui do estômago para o esôfago e órgãos adjacentes. Esse processo, associado a fatores ambientais, leva à presença de sintomas esofágicos e extra-esofágicos como regurgitação, tosse, odinofagia e pirose, sendo esse o principal sintoma responsável por interferir nas atividades diárias e diminuir a qualidade de vida do paciente (NWOKEDIUKO SC, et al., 2020).

A fisiopatologia dessa condição ainda não está completamente definida, mas pode ser simplificada como o mau funcionamento do esfíncter esofágico inferior, gerando os sintomas, principalmente, quando o indivíduo se encontra em decúbito e/ou após a realização de refeições. Apesar disso, sabe-se que a DRGE possui associação íntima com outras diferentes condições, tais como obesidade, hérnia de hiato e tabagismo, podendo gerar complicações como esôfago de Barrett e adenocarcinoma de esôfago (SCIDA S, et al., 2018).

Considerando sua prevalência, muitas pesquisas foram desenvolvidas sobre a DRGE, sendo publicado, em 2006, o relatório do Consenso de Montreal, estudo global que visava padronizar a definição e classificações da DRGE, permitindo assim que a doença fosse melhor compreendida. Desde a publicação desse consenso, diversos trabalhos vêm sendo publicados no campo da gastroenterologia, a fim de buscarem uma melhor compreensão do processo fisiopatológico dessa patologia e suas correlações epidemiológicas, como uma possível associação com o Helicobacter pylori, que é outra causa prevalente de distúrbios digestivos (WORLD GASTROENTEROLOGY ORGANISATION (WGO), 2015).

O Helicobacter pylori, o primeiro carcinógeno bacteriano a ser reconhecido, se trata de uma bactéria Gram-negativa, de forma espiral, flagelada, sendo responsável por promover uma colonização natural que se desenvolve na camada mucosa protetora do revestimento gástrico (ANSARI SA, et al., 2018). Essa bactéria surgiu ao conhecimento da comunidade científica em 1984, ainda como um bacilo não-identificado, mas já associado à gastrite estomacal e úlcera péptica (KONTOURAS J, et al., 2019).

A associação da $H$. pylori com algumas patologias ainda requer esclarecimento, contudo vários estudos apontam para a existência de sua influência na DRGE, sugerindo a ideia de um papel defensor dessa bactéria no desenvolvimento da patologia e suscitando, portanto, um possível benefício de tal infecção bacteriana. Por outro lado, com a compreensão das alterações que tal micro-organismo produz na secreção do ácido gástrico humano, também se levanta a possibilidade deste patógeno agravar os sintomas da DRGE, podendo levar a algumas complicações, se erradicada (LIU L, et al., 2018).

Por fim, a independência das duas entidades também é considerada no meio científico devido aos resultados de alguns estudos em anos recentes, levando ao entendimento de que a infecção por $H$. pylori e sua erradicação não possuem efeito na DRGE (COELHO LGV, et al., 2018). Assim, reconhecendo a complexidade desse cenário e a importância do conhecimento de uma doença para o manejo eficaz, essa breve revisão de literatura possui como objetivo discutir sobre a possível influência do Helicobacter pylori como um fator determinante na doença do refluxo gastroesofágico e suas possíveis complicações. 


\section{MÉTODOS}

Trata-se de uma revisão integrativa de literatura, voltada para os aspectos qualitativos do tema proposto, buscando analisar a influência da erradicação do $H$. pylori na DRGE. Por meio da busca bibliográfica nas bases de dados National Center for Biotechnology Information (NCBI), PubMed e Scientific Electronic Library Online (SciElo), foram utilizadas as palavras-chave, incluídas nos Descritores em Ciências de Saúde (DeCS), "Helicobacter pylori", "refluxo gastroesofágico", "esfíncter esofágico inferior", para identificar e incluir os estudos utilizados nesta revisão.

A pesquisa dos artigos foi realizada entre janeiro a agosto de 2021. Inicialmente, foram encontrados 230 artigos, porém apenas 15 destes se encontravam dentro dos critérios de elegibilidade. Os critérios de inclusão adotados para esse estudo foram: abordagem sobre DRGE e sobre $H$. pylori, em português ou inglês. Como critérios de exclusão, adotou-se não utilizar artigos com informações incompletas, artigos duplicados e estudos fora do período determinado (artigos publicados nos últimos 5 anos) e irrelevantes para a pesquisa (Figura 1).

Figura 1 - Fluxograma descrevendo os critérios de inclusão e exclusão adotados para o estudo.

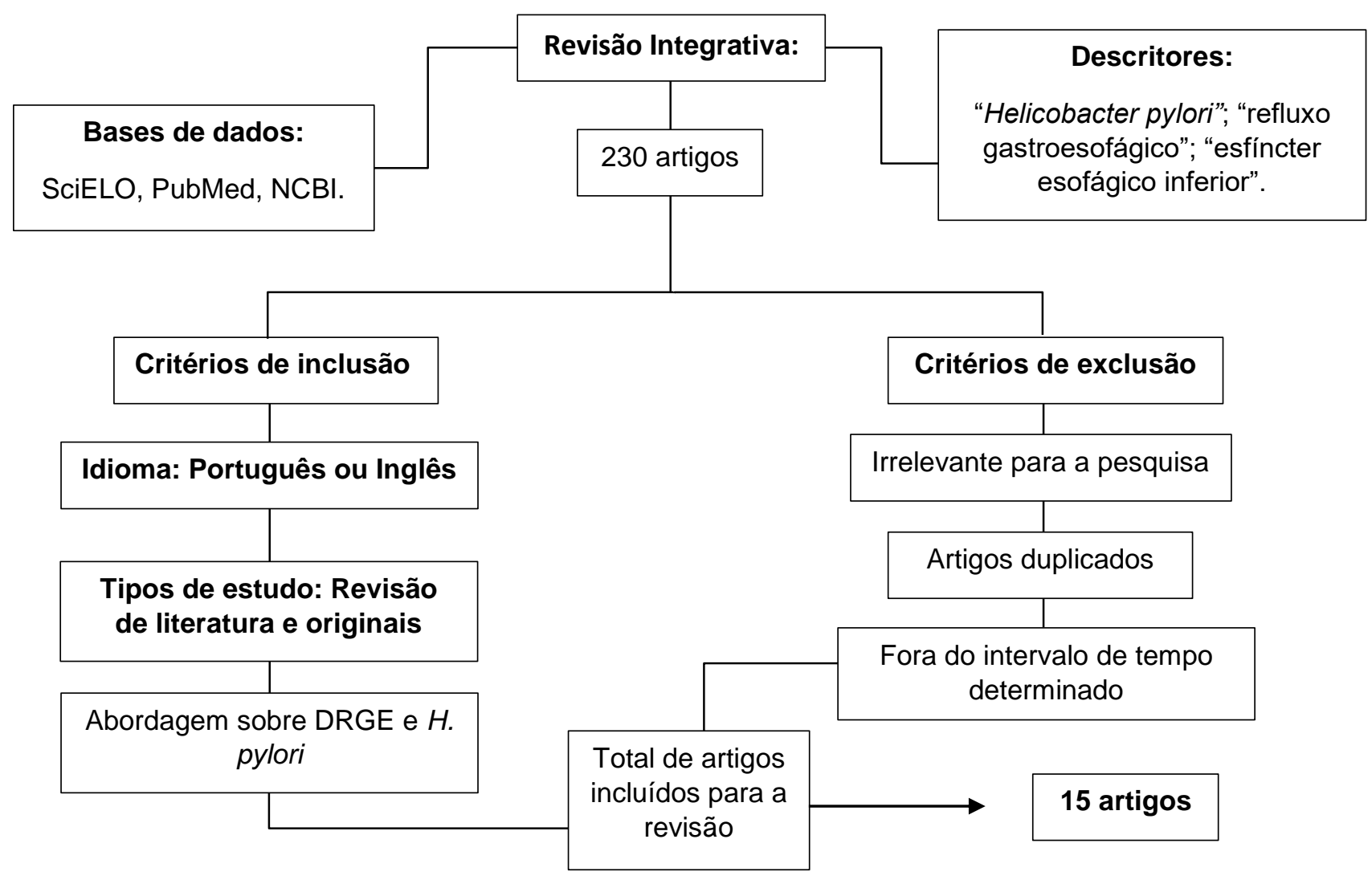

Fonte: Souza ALC e Amorim IFC, 2021.

\section{RESULTADOS}

Dentre os 15 artigos selecionados, a maioria diz respeito à relação entre a terapia de erradicação e a DRGE. Destes, cinco artigos apontam o H. pylori e a terapia de erradicação como fator protetor quanto ao desenvolvimento de sintomas de refluxo. Além disso, quatro artigos abordam a infecção bacteriana e seu tratamento como fatores de piora de DRGE, enquanto dois artigos tratam das duas doenças como coincidentes, sendo outros fatores relacionados ao desenvolvimento de doenças gastroesofágicas (Quadro 1). 
Quadro 1 - Síntese e caracterização dos principais achados dos estudos científicos incluídos na revisão.

\begin{tabular}{|c|c|c|c|}
\hline $\mathbf{N}$ & Autores (Ano) & Título & Tipo de Estudo/Principais Achados \\
\hline 1 & LIU L, et al (2018) & $\begin{array}{l}\text { Comparação de testes de função esofágica para } \\
\text { investigar o efeito da infeçãão por Helicobacter pylori } \\
\text { na Doença do Refluxo Gastroesofágico (DRGE). }\end{array}$ & $\begin{array}{l}\text { Estudo retrospectivo. Demonstra que participantes infectados por H. pylori } \\
\text { demonstraram melhor peristalse esofágica, aumento da pressão do } \\
\text { esfíncter esofágico inferior e redução da exposição do ácido sobre o } \\
\text { esôfago, indicando efeito protetor da bactéria sobre a DRGE. }\end{array}$ \\
\hline 2 & $\begin{array}{l}\text { KOUNTOURAS J, et al. } \\
\qquad(2019)\end{array}$ & $\begin{array}{l}\text { Uma perspectiva dos fatores de risco para } \\
\text { adenocarcinoma de esôfago: ênfase na infecção por } \\
\text { Helicobacter pylori. }\end{array}$ & $\begin{array}{l}\text { Revisão bibliográfica. Relata que a tentativa de amenizar o impacto da } \\
\text { DRGE através da manutenção do } H \text {. pylori aumenta o risco de } \\
\text { desenvolver neoplasias gastrointestinais, incluindo adenocarcinoma de } \\
\text { esôfago. }\end{array}$ \\
\hline 3 & ANSARI SA, et al. (2018) & $\begin{array}{l}\text { Associação de Helicobacter pylori oral com } \\
\text { complicações gástricas. }\end{array}$ & $\begin{array}{l}\text { Estudo de caso-controle. Apresenta que a infecção oral por Helicobacter } \\
\text { pylori elevou as chances de refluxo gastroesofágico grau II. }\end{array}$ \\
\hline 4 & $\begin{array}{l}\text { EL-ZIMAITY H, et al. } \\
\qquad(2018)\end{array}$ & $\begin{array}{l}\text { Fatores de risco para câncer de esôfago: ênfase em } \\
\text { agentes infecciosos. }\end{array}$ & $\begin{array}{l}\text { Revisão bibliográfica. Avalia que a redução da prevalência do } \\
\text { Helicobacter pylori associado ao aumento da incidência do } \\
\text { adenocarcinoma de esôfago pode ser uma coincidência e não uma } \\
\text { relação de causa e efeito. }\end{array}$ \\
\hline 5 & $\begin{array}{l}\text { NWOKEDIUKO SC, et al. } \\
\qquad(2020)\end{array}$ & $\begin{array}{l}\text { Doença do Refluxo Gastroesofágico em uma } \\
\text { população africana típica: um estudo multicêntrico } \\
\text { baseado em sintomas. }\end{array}$ & $\begin{array}{l}\text { Estudo observacional descritivo. Relata que numa região em que o } H \text {. } \\
\text { pylori é extremamente prevalente, a prevalência da DRGE é baixa, } \\
\text { associado também a uma baixa prevalência de obesidade. }\end{array}$ \\
\hline 6 & NA HK, et al. (2020) & $\begin{array}{l}\text { Efeito da erradicação do Helicobacter pylori na } \\
\text { esofagite de refluxo e nos sintomas da DRGE após } \\
\text { ressecção endoscópica de neoplasia gástrica: um } \\
\text { estudo prospectivo de centro único }\end{array}$ & $\begin{array}{l}\text { Estudo prospectivo. Evidencia que a erradicação do } H \text {. pylori não } \\
\text { aumentou a incidência dos sintomas de DRGE em pacientes que foram } \\
\text { submetidos à ressecção endoscópica gástrica. }\end{array}$ \\
\hline 7 & $\begin{array}{l}\text { ARGYROU A, et al. } \\
\qquad(2018)\end{array}$ & $\begin{array}{l}\text { Fatores de risco para Doença do Refluxo } \\
\text { Gastroesofágico e análise de contribuintes } \\
\text { genéticos. }\end{array}$ & $\begin{array}{l}\text { Revisão bibliográfica. Discute a combinação de genes que expressam } \\
\text { determinadas citocinas pró-inflamatórias e a infecção por } \mathrm{H} \text {. pylori, } \\
\text { levando a um possível efeito protetor contra a DRGE. }\end{array}$ \\
\hline 8 & $\begin{array}{l}\text { SUGIMOTO M, et al. } \\
\qquad(2021)\end{array}$ & $\begin{array}{l}\text { Risco de sintomas relacionados ao refluxo e } \\
\text { esofagite de refluxo após tratamento de erradicação } \\
\text { do Helicobacter pylori na população japonesa }\end{array}$ & $\begin{array}{l}\text { Estudo observacional retrospectivo. Demonstra que a terapia de } \\
\text { erradicação foi associada a uma menor prevalência de sintomas de } \\
\text { refluxo e dismotilidade. }\end{array}$ \\
\hline
\end{tabular}




\begin{tabular}{|c|c|c|c|}
\hline $\mathbf{N}$ & Autores (Ano) & Título & Tipo de Estudo/Principais Achados \\
\hline 9 & $\begin{array}{l}\text { SUGIMOTO M, et al. } \\
\qquad(2020)\end{array}$ & $\begin{array}{l}\text { Esofagite de refluxo endoscópico e sintomas } \\
\text { relacionados ao refluxo após terapia de erradicação } \\
\text { do Helicobacter pylori: meta-análise }\end{array}$ & $\begin{array}{l}\text { Meta-análise. Expôs que a terapia de erradicação foi associada a um } \\
\text { maior risco de desenvolver refluxo esofágico endoscópico, sendo esse } \\
\text { risco aumentado em países ocidentais, devido ao estilo de vida, fatores } \\
\text { genéticos populacionais e virulência das cepas de } H \text {. pylori. }\end{array}$ \\
\hline 10 & $\begin{array}{l}\text { MIFTAHUSSURUR M, et } \\
\text { al. (2018) }\end{array}$ & $\begin{array}{l}\text { Doença do Refluxo Gastroesofágico em uma área } \\
\text { com baixa prevalência de infecção por Helicobacter } \\
\text { pylori }\end{array}$ & $\begin{array}{l}\text { Estudo retrospectivo. Relata que numa região em que a infecção por } H \text {. } \\
\text { pylori possui baixa prevalência, a DRGE possui alta prevalência. Além } \\
\text { disso, maior poder aquisitivo e obesidade também foram sugeridas como } \\
\text { sendo fatores de risco adicionais no desenvolvimento da DRGE. }\end{array}$ \\
\hline 11 & LV J, et al. (2019) & $\begin{array}{l}\text { Alteração da microbiota esofágica no esôfago de } \\
\text { Barrett e adenocarcinoma esofágico. }\end{array}$ & $\begin{array}{l}\text { Revisão bibliográfica. Discute as alterações que a terapia da erradicação } \\
\text { do } H \text {. pylori poderiam acarretar no esôfago, podendo ocasionar o esôfago } \\
\text { de Barrett e o adenocarcinoma de esôfago. }\end{array}$ \\
\hline 12 & HARADA A, et al. (2019) & $\begin{array}{l}\text { Fatores de risco para esofagite de refluxo após } \\
\text { erradicação do Helicobacter pylori }\end{array}$ & $\begin{array}{l}\text { Estudo retrospectivo. Define o sexo masculino, índice de massa corporal } \\
25 \mathrm{~kg} / \mathrm{m}^{2} \text {, uso de bloqueadores de canal de cálcio e hérnia de hiato como } \\
\text { fatores de risco associados à esofagite de refluxo }\end{array}$ \\
\hline 13 & YUSEL O (2018) & $\begin{array}{l}\text { Interações entre Helicobacter pylori e doença do } \\
\text { refluxo gastroesofágico }\end{array}$ & $\begin{array}{l}\text { Estudo de Revisão. Afirma associação entre DRGE e HP de acordo com } \\
\text { localização no estômago, positividade de CagA e diferença geográfica. }\end{array}$ \\
\hline 14 & ZAMANI M, et al. (2021) & $\begin{array}{l}\text { Revisão sistemática com meta-análise: associação } \\
\text { de infecção por Helicobacter pylori com refluxo } \\
\text { gastroesofágico e suas complicações }\end{array}$ & $\begin{array}{l}\text { Revisão sistemática e meta-análise. Indica que a infecção por } \\
\text { Helicobacter pylori está associada com risco reduzido de sintomas } \\
\text { dispépticos e esofagite de refluxo, porém não apresenta risco aumentado } \\
\text { para desenvolvimento de esôfago de Barrett. }\end{array}$ \\
\hline 15 & ZHAO Y, et al. (2020) & $\begin{array}{l}\text { O efeito da erradicação do Helicobacter pylori em } \\
\text { pacientes com Doença do Refluxo Gastroesofágico: } \\
\text { uma meta-análise de estudos controlados } \\
\text { randomizados }\end{array}$ & $\begin{array}{l}\text { Revisão sistemática e meta-análise. Sugere que a erradicação do } \\
\text { Helicobacter pylori pode ocasionar o surgimento de DRGE, todavia não } \\
\text { possui impacto sobre pacientes em tratamento ou curados da doença } \\
\text { preexistente. }\end{array}$ \\
\hline
\end{tabular}

Fonte: Souza ALC e Amorim IFC, 2021. 


\section{DISCUSSÃO}

A doença do refluxo gastroesofágico é uma condição em que o conteúdo ácido estomacal reflui para o esôfago. Este órgão, por sua vez, atua por meio de ação peristáltica intermediando a passagem do alimento da faringe para o estômago, e, além disso, possui diversas proteções contra tais conteúdos ácidos que possam refluir. Diante disso, temos como consequência da quebra desse mecanismo fisiológico de proteção a ocorrência do refluxo em seu aspecto patológico (SCIDA S, et al., 2018).

Nesse contexto, o aparecimento de sintomas sugestivos e frequentes de pirose, associados a outros fatores epidemiológicos, como obesidade ou tabagismo, deve chamar a atenção para a DRGE. Diante desse quadro altamente sugestivo, o uso empírico de fármacos inibidores da bomba de próton (IBPs) pode servir como prova terapêutica que endossam fortemente o diagnóstico dessa condição (HENRY MACA, 2014).

Entretanto, em pacientes refratários à terapia empírica ou que possuam sinais de alarme, como disfagia ou perda abrupta de peso, reserva-se a indicação de exames específicos a fim de uma melhor investigação do quadro. Os principais exames considerados, neste caso, são a pHmetria de $24 \mathrm{~h}$, manometria do esôfago e a endoscopia digestiva alta, buscando visualizar possíveis complicações da DRGE, como a esofagite erosiva, esôfago de Barret e adenocarcinoma de esôfago (LIU L, et al., 2018). Essas condições também estão em ascensão nos países ocidentais, o que justifica a necessidade de buscar por possíveis fatores protetores, a fim de evitar a incidência de tais complicações decorrentes da DRGE (KOUNTOURAS J, et al, 2019).

O surgimento da doença do refluxo gastroesofágico e suas possíveis complicações dependem de fatores como o funcionamento da barreira antirrefluxo que é composta pelo esfíncter inferior do esôfago e pela porção crural do diafragma. Para mais, do conteúdo ácido estomacal, da resistência intrínseca do epitélio esofagiano, dos mecanismos de depuração intraluminal esofagianos e da duração da exposição do esôfago ao ácido. Esses fatores, em um funcionamento indevido, podem levar ao surgimento de lesões na mucosa distal do esôfago, o que pode ser demonstrado na endoscopia através da esofagite erosiva ou do esôfago de Barrett (SCIDA S, et al., 2018).

O Helicobacter pylori permanece sendo um agente infeccioso de alta prevalência, especialmente em populações que possuem baixas condições socioeconômicas, em que a transmissão ocorre de pessoa para pessoa. Tal patógeno pode estar relacionado à diversas manifestações gástricas e extra-gástricas devido a associação de algumas determinantes fisiopatológicas que envolvem, desde o sítio de implantação da bactéria, à falha de mecanismos celulares e imunológicos que deveriam proteger contra as manifestações desse patógeno (TOSCANO EP, et al., 2018).

A bactéria é microbiologicamente adaptada a colonizar somente a mucosa gástrica. Mesmo quando encontrada em porções anatômicas, a exemplo do duodeno, a sua colonização se limita às ilhas de metaplasia gástrica, onde o tecido do intestino duodenal é substituído por um tecido gástrico. As proteínas do bacilo induzem a formação de citocinas pro-inflamatórias que estimulam a quimiotaxia de outros neutrófilos e linfócitos, podendo gerar um desequilíbrio na barreira mucosa desse sítio (LV J, et al., 2019). A combinação dessas propriedades em um paciente suscetível pode acabar levando-o a desenvolver um quadro de gastrite crônica, porém, de modo geral, a infecção aguda pela bactéria não causa repercussões sintomáticas em nenhum indivíduo (ARSLAN N, et al., 2018).

A infecção por $H$. pylori pode estar associada com pelo menos dois padrões de variação da acidez estomacal. Na maioria dos casos, a infecção se dá isoladamente na região antral do estômago (o último terço distal do órgão), o que ocorre através da ativação das células $\mathrm{G}$, histologicamente específicas dessa localização. Com isso, a bactéria pode estimular a secreção da gastrina, peptídeo que atua na secreção do ácido gástrico estomacal e também atua na motilidade gástrica, levando o indivíduo ao estado de hipercloridria (EL-ZIMAITY H, et al., 2018).

A primeira linha da terapia de erradicação do $H$. pylori em países ocidentais consiste no uso de Inibidores da Bomba de Prótons (IBPs), claritromicina e azitromicina, por um período de 7 a 14 dias. Trata- 
se de uma associação tripla bem tolerada e com eficácia comprovada perante a maioria dos pacientes. Como alternativa, especialmente para pacientes refratários, pode ser instituída a terapia quadrupla baseada em bismuto, que consiste na combinação de metronidazol, tetraciclinas, IBPs e bismuto, também durante 7 a 14 dias (MALFERTHEINER P, et al., 2016).

Por seu caráter restrito ao trato digestivo, em especial às células estomacais, muitas pesquisas foram desenvolvidas buscando uma associação entre essa bactéria e o surgimento da esofagite de refluxo, o que poderia estar relacionado com a DRGE. O Helicobacter pylori, portanto, poderia atuar de diversas formas: alterando a secreção ácida estomacal; diminuindo a pressão no esfíncter esofágico e agindo diretamente sobre a mucosa do esôfago ou indiretamente, através de substâncias tóxicas secretadas pelo próprio organismo em resposta ao refluxo estabelecido (SUGIMOTO M, et al., 2020).

Contudo, é questionável se a presença da bactéria, independente das suas consequências para o ácido estomacal, seria o suficiente para atestar uma possível influência negativa sobre a fisiologia gastroesofágica. Um estudo realizado em 2018 demonstrou que a existência de $H$. pylori oral, por si só, acarretaria em maior risco de desenvolver DRGE, além de ocasionar frouxidão nos esfíncteres esofágicos. Além disso, é ponderado que manifestação de cepas do $H$. pylori na mucosa oral seria simplesmente o primeiro sítio de localização da bactéria que posteriormente ganhariam o trato gastrointestinal (ANSARI SA, et al., 2018).

Por outro lado, uma minoria dos indivíduos infectados pela $H$. pylori, quando acometidos nas regiões do corpo ou fundo gástrico, os dois terços proximais do órgão, acabam desenvolvendo um segundo padrão fisiopatológico, caracterizado por gastrite difusa ou atrófica, com consequente redução da produção e secreção do ácido clorídrico, visto que as células oxínticas, responsáveis por essas ações, se encontram nesta região estomacal. Como o ácido gástrico atuaria na inibição da liberação da gastrina pelas células $\mathrm{G}$, por feedback, o resultado da hipocloridria ou acloridria estomacal é uma hipergastrinemia (SUGIMOTO M, et al., 2020).

O efeito da hipergastrinemia na mucosa gástrica relaciona-se com o surgimento de tumores carcinoides gástricos. Todavia, no contexto da DRGE, enfatiza-se o papel da hipocloridria, isto é, se a partir da diminuição na secreção do conteúdo ácido, o quadro erosivo na porção distal do esôfago poderia se atenuar (EL-ZIMAITY H, et al., 2018). Supõe-se que, nesses pacientes com pangastrite hipoclorídrica, ao erradicar o bacilo ao tentar normalizar a secreção gástrica à níveis próximos ao basal, o paciente poderia manifestar ou até mesmo exacerbar sintomas característicos da DRGE (SUGIMOTO M, et al., 2020).

Um retrospectivo recente trouxe dados que corroboram com o papel protetor do $H$. pylori no contexto da DRGE. Utilizando dados de 124 pacientes diagnosticados com DRGE e submetidos a exames para avaliação do esôfago, os dados demonstraram que a peristalse esofágica era melhor nos indivíduos $H$. pylori-positivos, bem como a pressão no esfíncter esofágico inferior era maior. Além disso, a exposição esofágica ao ácido era reduzida, sugerindo tal efeito protetor sobre a mucosa esofágica. Contudo, tal estudo é considerado fraco por falta de amostra populacional com positividade para $H$. pylori pelo teste rápido de urease (LIU L, et al, 2018).

O papel da influência genética no desenvolvimento da DRGE também foi levantado como uma possível justificativa para determinar o papel maléfico ou benéfico do $H$. pylori. De acordo a Argyrou A, et al. (2018), a combinação de citocinas pró-inflamatórias expressas por determinados genes (IL-1B and IL-1RN) quando combinadas à infecção por Helicobacter pylori, poderiam exercer um certo efeito protetor contra 0 desenvolvimento de DRGE devido à potencialização da destruição de células parietais.

Por outro lado, aponta-se que a variação genética do próprio bacilo pode influenciar em seu potencial efeito nocivo ou protetor no indivíduo. Foi estabelecido que a cepa da bactéria que contém o gene cagA possui um fator de virulência importante, suscetibilizando o indivíduo infectado à úlcera péptica e aumentando o risco para adenocarcinoma gástrico. Essas cepas também estão associadas à uma maior indução de citocinas, influenciando em uma maior agressão celular (ANSARI SA, et al., 2018; TOSCANO EP, et al., 2018; SUGIMOTO M, et al., 2020). 
Outro fator a ser pontuado seria a alteração da composição da microbiota esofágica após o uso da terapia de erradicação. Tais alterações nesse contexto exerceriam um possível aumento dos níveis séricos de grelina, que, eventualmente, poderiam acarretar em maior susceptibilidade ao desenvolvimento de obesidade, gerando consequências na dinâmica do esvaziamento gástrico e promovendo o desenvolvimento de patologias como esôfago de Barret e neoplasia esofágica (LV J, et al., 2019).

Ainda que houvesse um papel protetor proveniente do $H$. pylori, é válido ressaltar que é conhecida a ação carcinogênica dessa bactéria na carcinogênese de outros sistemas. Segundo Kontouras J, et al. (2019), o H. pylori é um agente determinante nas neoplasias gástrica e colorretal, logo, parece inconsistente que sua relação com a DRGE e suas possíveis complicações seja benéfica ou protetora. Por fim, os autores destacam que a própria patogênese da DRGE torna difícil isolar apenas um fator de risco (como a infecção por $H$. pylori) sem considerar os outros, o que pode ser um dos motivos da discordância nos resultados presentes em diversos estudos ao longo do tempo.

Em outro estudo, El-Zimaity $\mathrm{H}$, et al. (2018) relatam que podem haver dois processos simultâneos que levariam à falsa crença de que o $\mathrm{H}$. pylori atua como fator protetor na DRGE: inicialmente, o aumento da incidência do adenocarcinoma de esôfago e do esôfago de Barrett associada à redução da prevalência do Helicobacter pylori pode ser uma mera coincidência e esses eventos não possuem relação de causa e efeito. Além disso, há um aumento na prevalência de obesidade, o que pode causar uma redução na pressão do esfíncter esofágico inferior, tornando o indivíduo mais suscetível ao desenvolvimento da doença do refluxo gastroesofágico.

Pensando ainda no desenvolvimento de complicações, uma revisão sistemática feita por Zamani M, et al. (2021) demonstrou que a infecção por $H$. pylori parece se associar com redução dos riscos de sintomas dispépticos e esofagite de refluxo. Contudo, em pacientes com DRGE já estabelecida, a infecção por $H$. pylori não parece aumentar os riscos para esôfago de Barrett. Além disso, a pesquisa pontua que tais possíveis efeitos protetores da infecção por esse patógeno não foram observados em estudos do tipo casocontrole.

Observou-se em alguns estudos que, após a erradicação bacteriana, alguns dos pacientes desenvolvem sintomas da DRGE. Contudo, não se sabe se o surgimento da doença está, de fato, relacionado com a erradicação da bactéria. Sugimoto $\mathrm{M}$, et al. (2021), em estudo observacional retrospectivo, associaram a terapia de erradicação à menor prevalência de sintomas de refluxo e dismotilidade, enquanto não houve alterações significativas dos sintomas em pacientes com falha terapêutica. Os autores relatam que, após a terapia de erradicação efetiva, os sintomas relacionados ao refluxo não sofreram redução significativa em pacientes $H$. pylori positivos com DRGE, porém melhoraram em pacientes com a infecção e sem DRGE, considerando-se, assim, a maioria desses sintomas gastroesofágicos como relacionados ao $\mathrm{H}$. pylori.

Em um outro estudo, dessa vez uma meta-análise que comparava a incidência de refluxo esofágico endoscópico de repetição e sintomas relacionados ao refluxo, a terapia de erradicação foi associada ao maior risco de desenvolvimento de esofagite de refluxo, sendo esse risco aumentado em países ocidentais, devido ao estilo de vida, fatores genéticos populacionais e virulência das cepas de $H$. pylori. Considerando que a terapia de erradicação corrige $\mathrm{opH}$ estomacal, o estudo considera que $\mathrm{o}$ grau de gastrite durante a erradicação influencia na recuperação da secreção ácida e o consequente risco de desenvolvimento de refluxo (SUGIMOTO M, et al., 2020).

De maneira similar, Harada A, et al. (2019) dissertaram sobre a existência de fatores de risco que levam a permanência da esofagite de refluxo mesmo após erradicação da bactéria em questão. Muito do que foi levantado pelos autores corrobora com o que se apresenta na maioria dos estudos sobre o tema, pois novamente se estabelece o sexo masculino, valores de IMC característicos de obesidade leve, tabagismo e hérnia de hiato e uso de bloqueadores do canal de cálcio como fatores de risco mesmo que haja a erradicação da $H$. pylori em um período de 4 a 24 meses. Para mais, Zhao Y, et al. (2020) avaliaram que a erradicação do $H$. pylori pode acarretar em DRGE, porém a terapia não gera impacto nas taxas de cura ou recidiva de pacientes com DRGE preexistente. 
Em contrapartida, diversos autores têm publicado que a erradicação do $H$. pylori não exerce influência na doença do refluxo gastroesofágico, baseado na realização de diversos ensaios clínicos. Na HK, et al. (2020) realizaram um estudo prospectivo em pacientes $H$. pylori positivos que foram submetidos à ressecção endoscópica do estômago. Após 4 semanas deste procedimento, iniciaram terapia para erradicação do Helicobacter pylori, e em seguida, foram acompanhados ao longo de 6, 12 e 18 a 24 meses. Durante esse período, não houve diferença significativa no desenvolvimento de sintomas típicos da DRGE entre os indivíduos $H$. pylori positivos (refratários a terapia de erradicação) e $H$. pylori negativos.

Desse modo, supõe-se que tanto o agravamento dos sintomas quanto o seu abrandamento, devido a possíveis efeitos protetores, não teriam relação com a simples decisão de erradicar a bactéria. Nesta perspectiva, a correlação inversa entre infecção por $H$. pylori e a prevalência ou severidade da DRGE, anteriormente citada, pode ser explicada pelos fatores demográficos que estão em constantes mudanças ao redor do globo, em especial, aumento nos níveis socioeconômicos (SUGIMOTO M, et al., 2020).

Comparando a DRGE em regiões com baixa e alta prevalência de $H$. pylori, estudos associando as duas patologias considerando a diferença geográfica demonstram que no Ocidente a terapia de erradicação está associada a maior risco de DRGE do que na Ásia, podendo, assim, a proteção estar presente apenas na população asiática. Apesar disso, devido às incertezas, os sintomas de DRGE não devem impedir 0 tratamento de H. pylori (YUSEL O, 2018).

De forma específica, um estudo conduzido na Indonésia que avaliou pacientes com sintomas de dispepsia, porém sendo a maioria $(98,1 \%) \mathrm{H}$. pylori negativos, concluiu que fatores como tabagismo e uso de IBP's significavam risco aumentado de desenvolver a doença do refluxo gastroesofágico. Além disso, a obesidade e o IMC elevado, associados à melhores condições econômicas também foram sugeridas como sendo determinantes no desenvolvimento da DRGE (MIFTAHUSSURUR M, et al., 2018).

Na Nigéria, de modo contrário, avaliou 3520 pacientes, dos quais 376 preenchiam critério diagnóstico para DRGE, e concluiu que a prevalência desta condição nessa população é inferior se comparada à prevalência norte Americana ou Europa Ocidental. O estudo também menciona que o país notadamente possui alta prevalência para Helicobacter pylori, e adicionalmente, possui uma baixa prevalência de obesidade (NWOKEDIUKO SC, et al., 2020).

É válido ressaltar que, atualmente, há uma diminuição progressiva na prevalência global da infecção por H. pylori, atribuída basicamente às melhoras de condições de higiene e saneamento, o que acarreta em menores riscos de transmissão do bacilo. Ao mesmo passo, a prevalência da obesidade e do sedentarismo, associados as demais alterações nos hábitos alimentares podem refletir no aumento da incidência das doenças associadas ao refluxo patológico (WGO, 2015).

Por conta disso, de acordo ao último documento publicado pelo World Gastroenterology Organisation (2015) sobre a DRGE, a terapia para erradicar o H. pylori deve ser instituída na grande maioria dos pacientes, devido ao potencial risco de carcinogênese somente pelo fato de albergar tal bactéria, acarretando em uma doença que tende a ser muito mais grave e letal para esses pacientes de modo geral. Por fim, segundo o relatório do Quinto Consenso de Maastricht/Florença (2017), na vigência deste manejo terapêutico, não há grande relevância clínica nas alterações da produção do ácido gástrico na grande maioria da população. (MALFERTHEINER P, et al., 2016).

\section{CONSIDERAÇÕES FINAIS}

Diante da sua prevalência e influência na qualidade de vida dos indivíduos, a DRGE é um tópico globalmente discutido, bem como sua relação com aspectos epidemiológicos e fisiopatológicos. Nessa perspectiva, sua associação com Helicobacter pylori é algo que gera dúvidas na comunidade científica, sendo alvo constante de estudos e pesquisas. Apesar da literatura biomédica nos fornecer trabalhos relevantes e significativos a respeito do tema, ainda não há um consenso bem estabelecido sobre o papel desse patógeno como um fator determinante na DRGE, sendo necessário, portanto, mais estudos que consigam definir de maneira clara a influência da Helicobacter pylori na DRGE, especialmente na população Ocidental, para que dessa forma, medidas terapêuticas nesse cenário sejam melhor otimizadas e individualizadas em benefício dos pacientes. 


\section{REFERÊNCIAS}

1. ANSARI SA, et al. Association of oral Helicobacter pylori with gastric complications. Life Sciences, 2018; 205: 125130.

2. ARGYROU A, et al. Risk factors for gastroesophageal reflux disease and analysis of genetic contributors. World Journal Of Clinical Cases, 2018; 6(8): 176-182.

3. ARSLAN N, et al. Importance of antimicrobial susceptibility testing for the management of eradication in Helicobacter pylori infection. World Journal Of Gastroenterology, 2017; 23(16): 2854-2869.

4. COELHO LGV, et al. Ivth Brazilian Consensus Conference On Helicobacter Pylori Infection. Arq. Gastroenterol., 2020; 55(2): 97-121.

5. EL-ZIMAITY H, et al. Risk factors for esophageal cancer: emphasis on infectious agents. Annals Of The New York Academy Of Sciences, 2018; 1434(1): 319-332.

6. HARADA A, et al. Risk factors for reflux esophagitis after eradication of Helicobacter pylori. Scand J Gastroenterol, 2019; 54(10): 1183-1188.

7. HENRY MACA. Diagnóstico e tratamento da doença do refluxo gastroesofágico. Arquivos Brasileiros de Cirurgia Digestiva, 2014; $27(3):$ 210-215.

8. KOUNTOURAS J, et al. A perspective on risk factors for esophageal adenocarcinoma: emphasis on helicobacter pylori infection. Annals Of The New York Academy Of Sciences, 2019; 1452(1): 12-17.

9. LIU L, et al. Comparison of Esophageal Function Tests to Investigate the Effect of Helicobacter Pylori Infection on Gastroesophageal Reflux Disease (GERD). Medical Science Monitor, 2018; 24: 4791-4797.

10. LV J, et al. Alteration of the esophageal microbiota in Barrett's esophagus and esophageal adenocarcinoma. World Journal Of Gastroenterology, 2019; 25(18): 2149-2161

11. MALFERTHEINER $P$, et al. Management of Helicobacter pylori infection - the Maastricht V/Florence Consensus Report. Gut, 2017; 66(1): 6-30.

12. MIFTAHUSSURUR M, et al. Gastroesophageal reflux disease in an area with low Helicobacter pylori infection prevalence. Plos One, 2018; 13(11): 1-15.

13. NA HK, et al. Effect of Helicobacter pylori eradication on reflux esophagitis and GERD symptoms after endoscopic resection of gastric neoplasm: a single-center prospective study. BMC gastroenterology, 2020; 20(1): 1-7.

14. NWOKEDIUKO SC, et al. Gastroesophageal reflux disease in a typical African population: a symptom-based multicenter study. BMC gastroenterology, 2020; 20(1): 1-8.

15. SCIDA S, et al. Relationship between Helicobacter pylori infection and GERD. Acta Bio Medica Atenei Parmensis, 2018; 89(8): 40-43.

16. SUGIMOTO M, et al. Risk of Reflux-Related Symptoms and Reflux Esophagitis after Helicobacter pylori Eradication Treatment in the Japanese Population. Journal Of Clinical Medicine, 2021; 10(7): 1-12.

17. SUGIMOTO M, et al. Endoscopic Reflux Esophagitis and Reflux-Related Symptoms after Helicobacter pylori Eradication Therapy: meta-analysis. Journal Of Clinical Medicine, 2020; 9(9): 1-18.

18. TOSCANO EP, et al. Epidemiological and Clinical-Pathological Aspects of Helicobacter pylori Infection in Brazilian Children and Adults. Gastroenterology Research And Practice, 2018; 2018: 1-8.

19. WORLD GASTROENTEROLOGY ORGANISATION (WGO). Perspectiva mundial sobre a doença do refluxo gastroesofágico: Global Guidelines. 2015. Disponível em: https://www.worldgastroenterology.org/guidelines/globalguidelines/gastroesophageal\%02reflux-disease/gastroesophageal-reflux-disease-gerd-portuguese. Acessado em: 01 de julho de 2021.

20. YUCEL O. Interactions between Helicobacter pylori and gastroesophageal reflux disease. Esophagus, 2019;16(1): 52-62.

21. ZAMANI M, et al. Systematic review with meta-analysis: association of Helicobacter pylori infection with gastrooesophageal reflux and its complications. Aliment Pharmacol Ther., 2021; 00:1-11.

22. ZHAO Y, et al. The Effect of Helicobacter pylori Eradication in Patients with Gastroesophageal Reflux Disease: A Meta-Analysis of Randomized Controlled Studies. Dig Dis., 2020; 38(4): 261-268. 